\title{
LOS ESTUDIANTES Y LA PROPAGACIÓN DE LAS SEÑALES MECÁNICAS: DIFICULTADES DE UNA SITUACIÓN DE VARIAS VARIABLES Y PROCEDIMIENTOS DE SIMPLIFICACIÓN
}

\author{
MAURINES, L. \\ I.D.P.E.S., París 7 Tour 23. 2, Place Jussieu. 75251 París Cedex 05. Francia.
}

\section{SUMMARY}

This paper analyses the general ways of reasoning used by students when learning the propagation of a signal on a string. Tendencies towards a reduction of the number of variables can be brought out. On the one hand, students tend to combine the variables into a single notion. Their reasoning seems to be based on the idea of a material object (here the bump on the string), described through this single notion. On the other hand, they consider simultaneously never more than two variables so that relations they have learned are changed into binary associations. From this analysis, some pedagogical implications are derived.

\section{INTRODUCCIÓN}

Este trabajo analiza las tendencias de conjunto de los razonamientos que los estudiantes ponen en práctica cuando abordan el estudio de la propagación de una señal transversal en una cuerda. Se manifiestan, particularmente estables, tanto antes de las lecciones sobre las ondas como mucho después.

En Francia el estudio de los fenómenos ondulatorios empieza en la clase que corresponde a tercero de BUP de ciencias, y se realiza mediante un enfoque esencialmente experimental, descriptivo y macroscópico. Se trata de presentar a los alumnos numerosos experimentos de propagación de señales variadas (en una cuerda, en un muelle, en el agua, con señales sonoras y luminosas), de mostrar en lo posible que la señal se propaga a una velocidad constante, de estudiar mediante gráficos (como los del cuadro I) las dos descripciones posibles de un fenómeno de propagación:

- la descripción espacial que da el estado del medio en un momento dado (fotografía de una señal en una cuerda);

- la descripción temporal que da la evolución del estado de un medio a lo largo del tiempo en un punto dado (gráfico del movimiento de un punto de la cuerda).

A este nivel de estudio, se confronta generalmente a los alumnos con:
Cuadro I

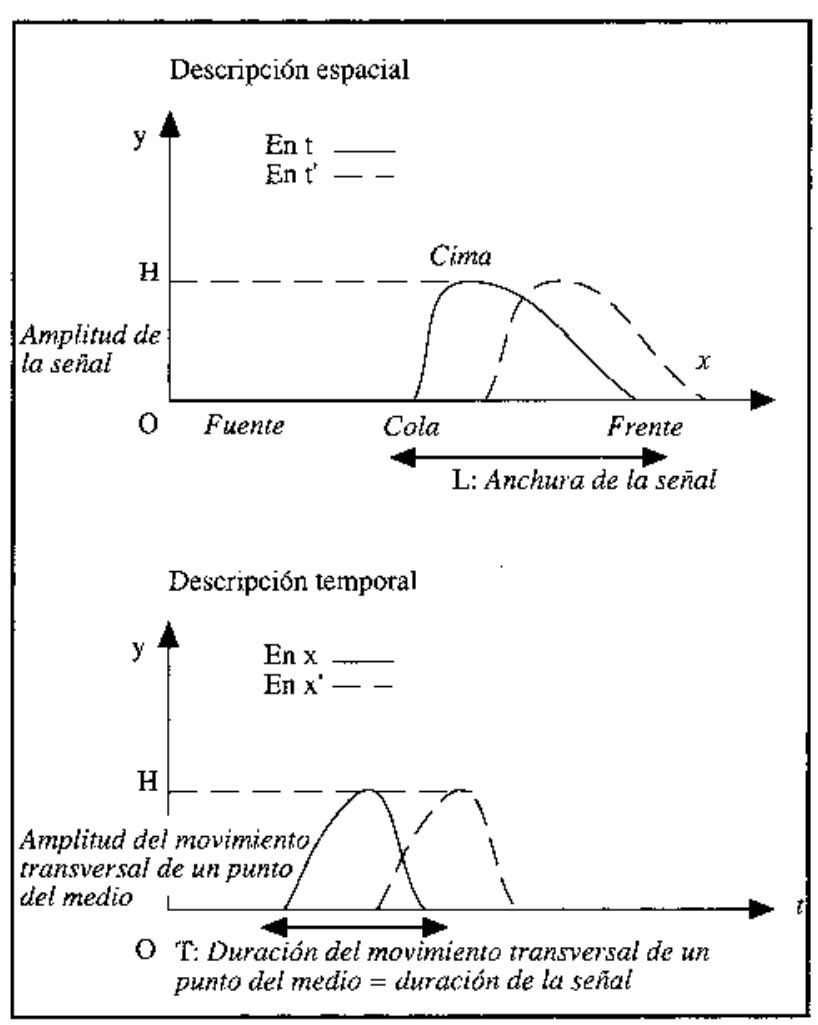


- el uso de distintas funciones de la variable tiempo, cuando la variable espacio está fijada $\left(\mathrm{f}_{x}(\mathrm{t})\right.$ para $\mathrm{x}$ y $\mathrm{x}^{\prime} \neq \mathrm{x}$ ); de distintas funciones de la variable espacio, cuando la variable tiempo está fijada $\left(\mathrm{gt}(\mathrm{x})\right.$ para $\mathrm{t}$ y $\left.\mathrm{t}^{\prime} \neq \mathrm{t}\right)$;

- tener que tomar en cuenta numerosas magnitudes físicas (velocidad de propagación $\mathrm{V}$, duración $\mathrm{T}$, anchura $\mathrm{L}$, amplitud $\mathrm{H}_{\text {...) }}$ que permiten caracterizar el fenómeno;

- la existenciade relaciones que hacen aparecer comomínimo tres magnitudes físicas, por ejemplo L=VT.

Ante las múltiples variables que están implicadas en el estudio de esta situación ondulatoria, los estudiantes ${ }^{1}$ ponen en práctica procedimientos de simplificación que pretenden reducir su número. Se puede destacar dos procedimientos:

- uno ligado a un razonamiento en términos de objeto: los estudiantes describen y explican la propagación de una señal en una cuerda focalizándose en la forma visible que se desplaza y considerando esa "protuberancia" como un objeto material fabricado y puesto en movimiento por la fuente, siendo el medio únicamente un soporte pasivo. Una sola noción, concepto híbrido y agrupador de magnitudes físicas ("el capital"), permite dar cuenta de esta "mecánica espontánea de la señal";

- otro que resulta de su incapacidad para considerar simultáneamente más de dos magnitudes físicas. Sólo se establecen dependencias funcionales entre dos magnitudes físicás.

Durante esta investigación se construyeron unos veinte cuestionarios. Ya que son demasiado numerosos para verlos todos en detalle, sólo damos integralmente el enunciado de algunos de ellos. Se pueden obtener los demás pidiéndolos al autor.

\section{EL CAPITAL, SOPORTE DEL RAZONA- MIENTO ESPONTANEO}

\section{La cuerda almacena un "capital" que la fuente le comunica y pone en movimiento}

Para introducir este capital, presentamos los resultados de una pregunta (Cuadro II) que trataba de ver si había una manera de mover la mano para que un punto de la culerda se moviera antes.

\begin{abstract}
A pesar de que la velocidad de propagación depende únicamente del medio, el $60 \%$ de los alumnos a los que se preguntó antes de que recibieran las clases $(\mathrm{N}=42)$ y el $75 \%$ después $(\mathrm{N}=16)$ contestaron que la velocidad de propagación depende de lo que hace la mano. Las numerosas justificaciones dinámicas indican que lo que importa es la fuerza que ejerce la mano para crear la señal. Cuanto mayor es, mayor es también la velocidad:
\end{abstract}

la protuberancia se desplazará cada vez más rápidamente si el gesto $^{2}$ es impetuoso,

la velocidad depende de la fuerza con la que se mueve la mano.

Se amalgama la causa ( la sacudida que da el experimentador) y el efecto (la protuberancia y su desplazamiento) todo parece suceder como si esta fuerza siguiera ligada a la señal y se desplazara con ella:

Cuadro II

Entunciado del cuestionario:

Se ata a una cuerda, en el punto $R$, un hilo rojo. Se coge con la mano un extremo de la cuerda, $O$.

$\mathbf{O}+\mathbf{R}$

Moviendo la mano, tenemos la forma siguiente en el momento t:

O.

$\mathbf{R}$

Pregunta: ¿Hay alguna mancra de mover la mano para que la forma alcance el hilo rojo antes?

Sí / No $/$ ¿Cuál?

Ejemplos de respuestas:

"...cuanto más impetuoso es el gesto, más deprisa irá la protuberancia"

"... se forman también ondas a causa de la fuerza $\vec{F}$ ".

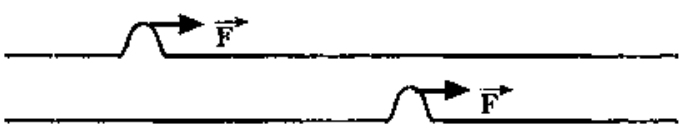


si la intensidad de la fuerza propagada es mayor, entonces la protuberancia se desplazará también más rápidamente.

Algunos de los esquemas proporcionados refuerzan esta hipótesis: la fuerza que viene representada en ellos se sitúa en la protuberancia (Cuadro II).

La fuente parece pues comunicar algo a la cuerda cuando se forma la señal, algo que se desplaza y que queda situado en la protuberancia. Los alumnos lo llaman a menudo "fuerza", pero es en realidad una noción híbrida, mezcla de fuerza, energía y velocidad. Se ha llamado capital a esta fuerza, que tiene poco que ver con el sentido clásico que le da la física, ya que la cuerda la almacena como si de un capital se tratase.

\section{El capital tiene una forma y la protuberancia la materializa}

Una pregunta que pide comparar la velocidad de tres señales de formas diferentes muestra que la fuente da forma al capital como un artesano que fabrica una vasija:

El $87 \%$ de los alumnos a los que se interrogó antes de las clases $(\mathrm{N}=93)$ y el $41 \%$ después de ellas $(\mathrm{N}=27)$ contestaron que las velocidades de las distintas señales pueden ser distintas.

Las numerosas justificaciones dinámicas que hacían intervenir la fuerza ejercida por la mano y comunicada a la señal, es decir el capital, muestra que la "protuberancia" lo materializa, que la forma de la señal (más exactamente su amplitud) y la velocidad de propagación varían en el mismo sentido que él:

la $C^{3}$ va más deprisa, ya que la fuerza proporcionada por el brazo del niño modifica la forma de la protuberancia y la velocidad. Asípues, a fuerza más intensa corresponde una forma de la protuberancia mayor y mayor será también la velocidad.

... esto depende de la fuerza con la que se ha hecho el movimiento. Esto se ve según el tamaño de la protuberancia. Es el reflejo de la fuerza que hace el niño para llegar a este resultado....

\section{El capital agrupa magnitudes físicas adherentes}

Los alumnos perciben el movimiento de la protuberancia y su existencia como dos facetas de un solo objeto, el capital. Cuando éste varía, a consecuencia de frotamientos, por ejemplo, las dos magnitudes físicas asociadas a esas dos facetas ( $\mathrm{V}$ y $\mathrm{H}$ ) varían simultáneamente y en el mismo sentido que él: son adherentes. Así, a una pregunta sobre si la velocidad de propagación de una protuberancia que desaparece antes de alcanzar la otra extremidad de la cuerda es constante a lo largo del tiempo, el $68 \%$ de los alumnos interrogados antes de las explicaciones $(\mathrm{N}=56)$ y el $55 \%$ después de ellas $(\mathrm{N}=42)$ contestan que disminuye, ya que para ellos una disminu- ción de amplitud indica una disminución del capital y, en consecuencia, de la velocidad de propagación:

la altura es menos ya que la acción de la mano se atenúa,

si la protuberancia desaparece, eso es debido a que la fuerza que lo 'hacía' desaparece. Durante este tiempo, la velocidad disminuye.

\section{Cuadro III}

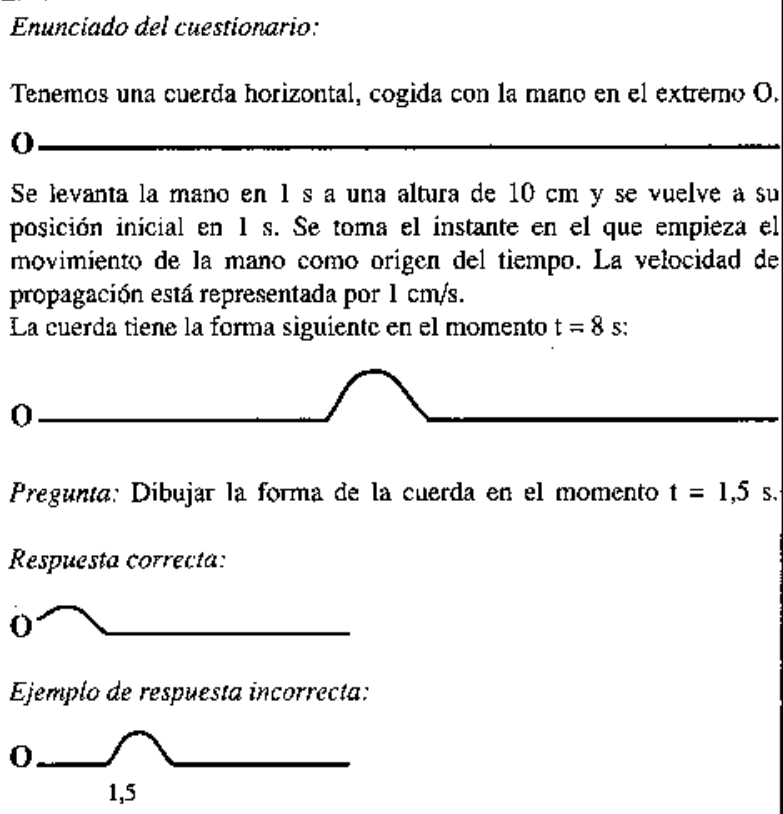

\section{El capital es un objeto ficticio fabricado por la fuente}

La forma del capital (y por lo tanto la de la protuberancia) tiene las mismas particularidades geométricas que las de un objeto material, a saber, de existir únicamente acabada y de no depender más que de la fuente.

Así, a una pregunta que pedía representat la cuerda antes que la mano volviera a su punto de partida (Cuadro III), el 53\% de los 47 estudiantes que se interrogó después de las explicaciones representa una señal completamente formada cuando en ese momento no lo está.

A una pregunta que les pide trazar en el mismo momento dos señales que se propagan sobre dos cuerdas distintas y obtenidas por movimientos de mano idénticos, el $60 \%$ de los 80 estudiantes interrogados después de la enseñanza representan señales de anchuras idénticas cuando deberían ser distintas, ya que la anchura $(\mathrm{L}=\mathrm{VT})$ de una señal depende no sólo de la fuente (por T) sino también del medio de propagación (por V).

Las justificaciones que proporcionan ponen de manifiesto que un razonamiento en términos del objeto puede llevar a los estudiantes a considerar un número restringido de magnitudes físicas por fijación implícita de una de ellas. En efecto, sólo hacen aparecer una asociación 
entre la anchura L y la duración $\mathrm{T}$, sin mencionar la velocidad V:

anchuras idénticas si damos exactamente la misma impuIsión $a C_{1}$ y $a C_{2}$,

$C_{1}=C_{2}$ la anchura del bulto depende de la duración de la sacudida inicial.

Este fenómeno se reproduce cuando, en la misma situación, se pide a los estudiantes que comparen la duración del movimiento de dos puntos de referencia situados sobre cada una de las cuerdas, a la misma distancia de los extremos. Aunque las duraciones de los dos movimientos son idénticas, el $48 \%$ de los 46 estudiantes interrogados después de la enseñanza dicen que el punto de referencia que se halla sobre la cuerda de mayor velocidad de propagación se mueve menos tiempo. Los estudiantes establecen aquí una asociación entre la duración $T$ y la velocidad $V$, siendo la anchura $L$ fija, y dan una respuesta que corresponde a lo que pasa cuando un ratón se mueve debajo de una alfombra: cuanto más deprisa va, menor es la duración del movimiento de un punto de la alfombra.

\section{Consecuencias de un razonamiento en términos de capital}

Mononocional, el razonamiento de los estudiantes se basa en la noción de capital. Este soporte les lleva a:

- reducir el número de variables, ya sea por su carácter agrupador de magnitudes físicas, que favorece una variación simultánea de variables adherentes (H disminuye cuando $V$ disminuye); ya sea por su carácter de objeto que sugiere la fijación implícita de una magnitud ( $\mathrm{L}$, la anchura de la señal, por ejemplo);

- aplicar a la protuberancia que se đesplaza una "mecánica" de objeto material (la velocidad de propagación de la señal depende de la fuente y puede variar a lo largo del tiempo) y caracterizar la señal por una magnitud geomé-

Cuadro IV

Enunciado del cuestionario:

Formulación cualitativa:

Dos cuerdas distintas están en el suclo. En cada una de las cuerdas se marca un punto Al para la cuerda $\mathrm{Cl}_{1} \mathrm{~A} 2$ para $\mathrm{C}_{2}$.

\begin{tabular}{|c|c|c|}
\hline & $A_{3}$ & Cuerda $C_{1}$ \\
\hline $\mathrm{O}_{2}$ & $\mathrm{~A}_{2}$ & Cuerda $\mathrm{C}_{2}$ \\
\hline
\end{tabular}

Situaciön A. Dos niños tienen respectivarnente en la mano el extremo $\mathrm{O}_{1}$ y $\mathrm{O}_{2}$ de las cuerdas. Cada uno hace un movimiento de mano enpezando a levantar la mano en el mismo momento y ven desplazarse en cada cuerda una "protuberancia". En el mismo momento ven:

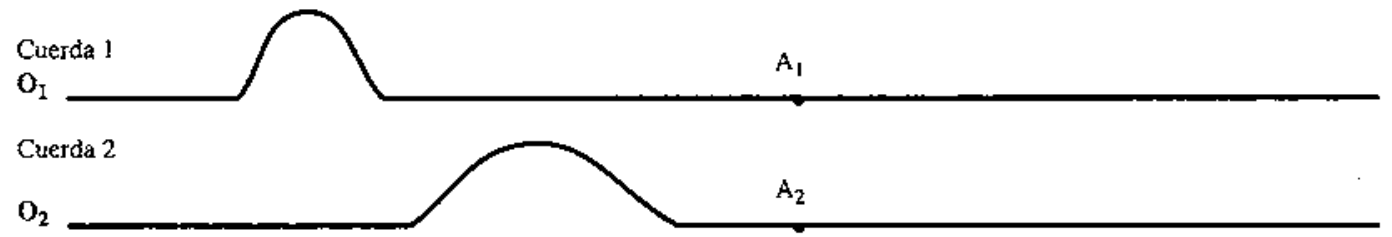

Pregunta: Comparar la duración del movimiento de Al y A2.

Justificar la respuesta.

Sinación B: Los niños hacen de nuevo el movimiento empezando a levantar la mano en el mismo momento y observan tas dos cuerdas en el mismo momento:

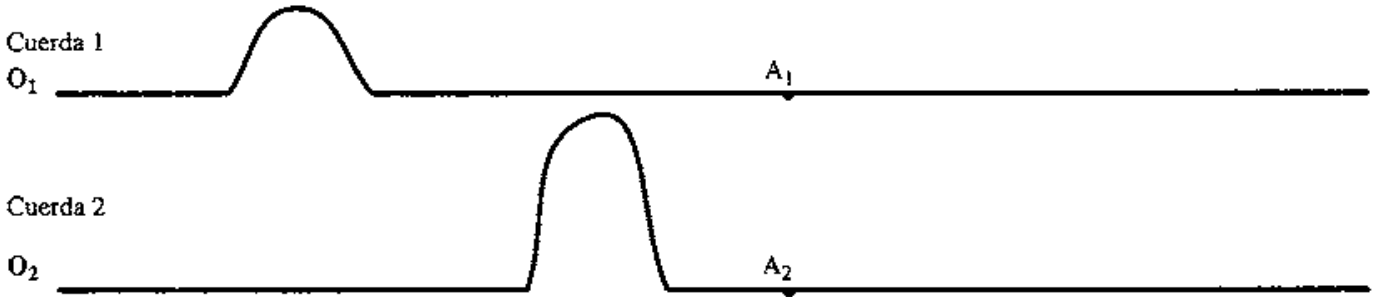

Pregunta: Comparar la duración del movimiento de Al y A2.

Justificar la respuesta.

Formulación cuantitativa: En esta version, se añadía los datos:

La celeridad Cı de la primera cuerda está representada por $1 \mathrm{~cm} / \mathrm{s}$ y la de la segunda cuerda por $2 \mathrm{~cm} / \mathrm{s}$. La escala de longitud es de $1 \mathrm{~cm} \longrightarrow 1 \mathrm{~cm}$. 


\section{Cuadro IV (Continuación)}

RESULTADOS: 37 estudiantes de los que recibieron explicaciones sobre las ondas respondieron a esas preguntas.

Situación A

\begin{tabular}{|l|c|c|}
\hline & $\begin{array}{c}\text { Respuesta correcta } \\
T_{1}=T_{3}\end{array}$ & $\begin{array}{c}\text { Respuesla inconcecta } \\
T_{1}<T_{2} \circ T_{1}>T_{2}\end{array}$ \\
\hline $\begin{array}{l}\text { Versión } \\
\text { cualicativa }\end{array}$ & $6 \%$ & $85 \%$ \\
\hline $\begin{array}{l}\text { Versión } \\
\text { cuanntitativ日 }\end{array}$ & $59 \%$ & $24 \%$ \\
\hline
\end{tabular}

Situación B

\begin{tabular}{|l|c|c|}
\hline & $\begin{array}{c}\text { Respuesta correcta } \\
\mathrm{T}_{2}<\mathrm{T}_{1}\end{array}$ & $\begin{array}{c}\text { Respuesta incorrecta } \\
\mathrm{T}_{2}>\mathrm{T}_{1} \circ \mathrm{T}_{1}=\mathrm{T}_{2}\end{array}$ \\
\hline $\begin{array}{l}\text { Version } \\
\text { cualitativa }\end{array}$ & $25 \%$ & $53 \%$ \\
\hline $\begin{array}{l}\text { Version } \\
\text { cuantitativa }\end{array}$ & $41 \%$ & $40 \%$ \\
\hline
\end{tabular}

trica (su anchura) y no temporal (su duración). La "mecánica espontánea de la señal" presenta semejanzas con la mecánica espontánea del sólido analizada por Viennot (1979) y Saltiel (1980);

- una interdependencia entre el movimiento transversal de un punto del medio y la velocidad de propagación (por ejemplo $\mathrm{H}$ y $\mathrm{V}$ varían simultáneamente).

\section{DEPENDENCIAS FUNCIONALES}

\section{Relación $\mathbf{l}=\mathrm{VT}$ y contenido numérico funcional}

Se puede ușar una relación como la de $\mathrm{L}=\mathrm{VT}$ para calcular el valor numérico de una de las magnitudes físicas mediante valores numéricos de las otras dos (puesta en práctica del contenido numérico) o para prever el sentido de variación de una de ellas conociendo el sentido de variación de las otras dos (puesta en práctica del contenido funcional).

El análisis de las respuestas obtenidas a un cuestionario (Cuadro IV), planteado sucesivamente de dos maneras (cualitativa y luego cuantitativa), a los mismos estudiantes que recibieron explicaciones sobre las ondas $(\mathrm{N}=$ 37), muestra claramente una tendencia a privilegiar el contenido numérico de una relación en detrimento del contenido funcional: la relación $\mathrm{L}=\mathrm{VT}$ es conocida, pero sólo se usa cuando se dan los valores numéricos. Las respuestas que proporcionan entonces los estudiantes son correctas en su mayoría. Hay que comparar esta tendencia a la "numerización" de las relaciones con la que apunta Viennot (1982) sobre las constantes: para los estudiantes esta palabra remite más a la idea de número que a la de función constante de varias variables.
Se plantea pues el problema de conocer las dependencias funcionales establecidas por los estudiantes en situaciones cualitativas. Vamos a ver a continuación qué les permite reducir el número de variables consideradas simultáneamente a dos.

\section{Asociaciones binarias}

Las respuestas que se obtienen a la formulación cualita tiva del cuestionario anterior (sean o no correctas) ponen de manifiesto que los alumnos establecen dependencias funcionales únicamente entre dos magnitudes físicas (aquélla sobre la que se basa la pregunta y otra magnitud pertienente o no), sin tomar en cuenta las demás.

Cuando hay que considerar una magnitud geométrica y una magnitud cinemática para comparar dos duraciones, Ios alumnos no tienen en cuenta más que una de ellas, sin mencionar la otra.

Así, en la situación $B$ :

- La respuesta incorrecta $\mathrm{T}_{1}<\mathrm{T}_{2}$ dada por el $50 \%$ de los alumnos se justifica en un $81 \%$ por $\mathrm{H}_{\mathrm{l}}<\mathrm{H}_{2}$ : Ios alumnos establecen una asociación no pertinente entre $\mathrm{H}$ y $\mathrm{T}$, y no mencionan ninguna velocidad.

- La respuesta correcta $T_{1}>T_{2}$ dada por el $25 \%$ de los alumnos se justifica en un $88 \%$ por $V_{1}<V_{2}$. Si los alumnos establecen aquí una asociación pertinente entre las dos magnitudes $V$ y $T$, no mencionan la tercera: Ia anchura $\mathrm{L}$.

La costumbre quiere que se diga, ante esta última respuesta, que la magnitud que no se menciona (L) está implícitamente fijada. Sin duda es mucho más apropiado decir que los alumnos no se ocupan de elia, ya que en los esquemas propuestos en la situación $A$, aunque se vea que las anchuras de las señales y las velocidades de propagación son distintas, los alumnos siguen mencionando únicamente dos magnitudes:

- La respuesta incorrecta $T_{1}<\Upsilon_{2}$ dada por el $66 \%$ de los alumnos se justifica en un $76 \%$ por $\mathrm{L}_{1}<\mathrm{L}_{2}$ : se establece una asociación pertinente entre $\mathrm{L}$ y $\mathrm{T}$ pero no se menciona ninguna velocidad.

- La respuesta incorrecta $T_{1}>T_{2}$ dada por el $19 \%$ de los alumnos se justifica en un $100 \%$ por $V_{1}<V_{2}$ : vueive a aparecer la asociación pertienente entre $\mathrm{V}$ y $T$, pero se sigue sin mencionar la anchura.

El uso funcional de las relaciones aprendidas (por ejemplo $\mathrm{L}=\mathrm{VT}$ ) es imposible, ya que precisa que se tome en cuenta simultáneamente todas las magnitudes que la constituyen (aquí tres). Esta imposibilidad se manifiesta claramente en los casos excepcionales en que los estudiantes se plantean dos asociaciones binarias contradictorias puesto que no pueden llegar a conclusiones. La respuesta siguiente, obtenida para la situación $\mathrm{A}$ del cuestionario anterior (Cuadro IV) lo indica claramente: 
Cuadro V

Enunciado del cuestionario:

Una cueróa está en el suelo. Se han marcado dos puntos A y B en esa cuerda.

$\mathbf{0}$

A

B

Un niño tiene en la mano el extremo $O$ de la cuerda. Hace un movimiento de mano y ve desplazarse en la cuerda una "protuberanciat". Observa la cuerda en 2 momentos distintos $t$ y $t$ ', $t$ ' $>t$ :
En t:
$\mathbf{O}$

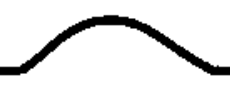
A

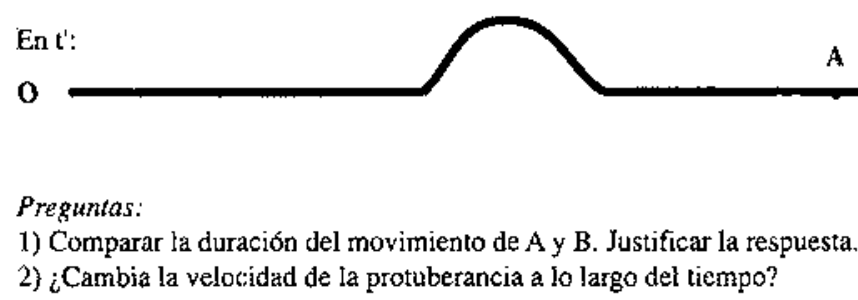

B

Respuestas:

1) El $69 \%$ de los 32 alumnos interrogados después de haber recibido clases contestan que las duraciones son iguales (lo que es correcto) y el $66 \%$ de ellos no mencionar la constancia de la velocidad de propagación.

2) El $59 \%$ de los que contestan correctamente a la primera pregunta contestan a ésta que la velocidad de propagación varia.

\section{Cuadro VI}

Tres cuerdas están en el suelo. En cada una se ha marcado un punto: $A_{1}$ para $C_{1}, A_{2}$ para $C_{2}, A_{3}$ para $C_{3}$.

$\mathrm{O}_{2} \longrightarrow$

Tres niños tienen respectivamente en là mano el extremo $\mathrm{O}_{1}, \mathrm{O}_{2}, \mathrm{O}_{3}$ de las cuerdas. Hacen cada uno un movimiento de mano empezando a lcvantar la mano en el mismo momento y ven desplazarse en cada cuerda una protuberancia. En un mismo instante:

Cuerda $C_{1}$

$\mathbf{O}_{\mathbf{k}}$

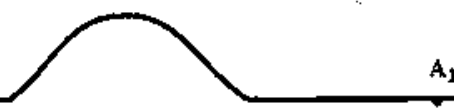

Cuerda $\mathrm{C}_{2}$

$\mathrm{O}_{2}$

$\mathbf{A}_{2}$

Cuerda $\mathrm{C}_{3}$

$\mathrm{O}_{3}$

$\mathbf{A}_{3}$

Pregunta: Comparar la duración del moviniento de $A_{1}, A_{2}, A_{3}$. Justificar la respuesta. 
la Jongitud de la señal es superior para $A_{2}$, por lo que $A_{2}$ estaría más tiempo en movimiento que $A_{1}$; pero $\mathrm{C}_{2}>$ $C_{1}, y$ la duración del movimiento es proporcional a $/ / C$ y a L, la longitud de la señal, por lo que no se pueden sacar conclusiones.

Aunque este tipo de razonamiento sea compatible con una respuesta correcta, esto no demuestra que los alumnos hayan entendido lo que sucede. En efecto, la comparación de las respuestas obtenidas a dos preguntas sobre la misma situación pone a menudo de manifiesto incoherencias: sucede que en una primera pregunta una magnitud parezca fijada implícitamente y en otra pregunta sea explícitamente variable (Cuadro V). Debe recalcarse que esta contradicción no sorprende a ningún estudiante: rara vez relacionan las respuestas de diversas preguntas.

Sucede a veces que aparecen incoherencias en la respuesta a una sola pregunta a consecuencia de un cambio de asociaciones. Basta con que en la situación propuesta haya al menos dos magnitudes distintas. Es el caso de la que presentamos en el cuadro VI: el $16 \%$ de los 62 alumnos interrogados antes de las explicaciones usan sucesivamente una asociación entre $\mathrm{L}$ y $\mathrm{T}$, y luego una asociación entre $\mathrm{H}$ y $\mathrm{T}$, y concluyen con la clasificación $\mathrm{T}_{\mathrm{A} 2}<\mathrm{T}_{\mathrm{A} 3}<\mathrm{T}_{\mathrm{A} 1}$

\section{la forma del movimiento es distinta pero la velocidad es} idéntica.

La anchura del movimiento de la cuerda l siendo mayor. $T_{A I} \geq T_{A 2} \geq T_{A 2}$ y como la altura es menor en $T_{2}$ que en $T_{3}$, la duración $T_{A 3}$ será mayor que $T_{A 2}$. Por lo tanto tenemos. $T_{A 2}<T_{A 3}<T_{A f}$.

\section{Existencia de encadenamientos de asociaciones binarias}

Cuando los alumnos deben considerar más de dos magnitudes físicas, las hacen intervenir encadenando asociaciones entre dos magnitudes, como en el ejemplo tan conocido que apuntaba Piaget (1972):

más deprisa $\rightarrow>$ más lejos, y más lejos $\rightarrow>$ más tiempo; por Io tanto: más deprisa $\rightarrow$ más tiempo.

Demos para empezar un ejemplo de encadenamiento obtenido en el cuestionario que pedía comparar la duración del movimiento transversal de tres puntos de referencia, situado cada uno en una cuerda (Cuadro VI):

tenemos $T_{A 3}<T_{A 2}<T_{A 1}$, ya que cuanto más alta es la protuberancia $\left(h_{1}<h_{2}<h_{3}\right)$, mayor es el trabajo proporcionado y, por lo tanto, la energía proporcionada, por lo que la velocidad de desplazamiento de la protuberancia a lo largo de la cuerda es mayor".

Aquí el alumno establece la cadena:

$\mathrm{H}$ aumenta $\rightarrow \mathrm{F}$ (es decir, el capital) aumenta $\rightarrow \mathrm{V}$ aumenta $\rightarrow \mathrm{T}$ disminuye.
Terminemos por esta respuesta obtenida a la primera pregunta del cuadro $V$ que pedía comparar la duración del movimiento transversal de dos puntos de referencia situados en la misma cuerda durante la propagación de una señal que disminuye de amplitud. Ilustra de modo ejemplar las contradicciones a las que pueden llegar los pocos alumnos que, transformando una relación en un encadenamiento de asociaciones binarias, desean especificar la tercera magnitud:

se observa que la onda se amortigua pero el período sigue siendo el mismo...

Sabemos que $\lambda=c T$, siendo $\lambda$ la longitud de onda.

$c=\lambda / T$, si la onda se amortigua (la velocidad de propagación disminuye 4 ) y si el período sigue idéntico, tendremos lógicamente $\lambda<\lambda$

tendremos pues que, considerando constante: $T_{A}>T_{B}$.

Por lo tanto, el alumno establece la cadena: $H$ disminuye $\rightarrow c$ disminuye $\rightarrow \lambda$ disminuye $\rightarrow \mathrm{T}$ disminuye.

Se contradice dos veces (una vez explícitamente hablando de T y otra implícitamente hablando de c) y contradice también el enunciado, ya que $\lambda$ es constante.

\section{CONCLUSIÓN Y SUGERENCIAS PEDA. GOGICAS}

La tendencia a reducir el número de variables que se observan en los estudiantes para situaciones de propagación no son espećfficas del campo ondulatorio. Otros trabajos (Closset 1989, Rozier 1990, Viennot 1988) ponen de manifiesto tendencias análogas en otros campos. Así pues, encontramos también un razonamiento mononocional en términos del objeto en electrocinética y en termodinámica: el objeto corresponde en ese caso a la "sustancialización" de un "bloque" de corriente o de calor y se puede introducir un "capital". El encadenamiento de asociaciones binarias que está en la base del razonamiento en termodinámica presenta en ese caso particular un carácter causal.

La enseñanza actual de las ondas no favorece el replanteamiento de estas tendencias a la numerización de las relaciones y a la reducción funcional. Se puede observar: un error encontrado en algunos manuales escolares (ya sea de enseñanza secundaria o superior) indica hasta qué punto estas tendencias pueden llegar a ser importantes y subsistir inciuso en los expertos. Constatamos en los esquemas de la página del cuadro VII que la señal incidente tiene la misma anchura que la señal transmitida.

Contradiciendo la serie de fotografías que sigue, pueden reforzar la asimilación señal-objeto y un razonamiento en términos de capital.

- Ocurre lo mismo con un dispositivo de simulación propuesto para estudiar la descripción espacial y tempo- 


\section{Cuadro VII}

Păgina extraida de un martual de Física (Alonson y Finn).

(a)

(b) $m_{1}>m_{2}$

(b) $m_{1}<m_{2}$

(1) Complementos a las teyes de refiextin y refracción

(1)

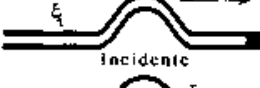

(1)

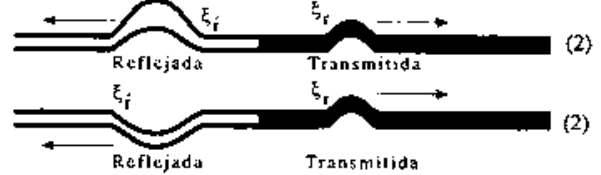

(d)

(e)
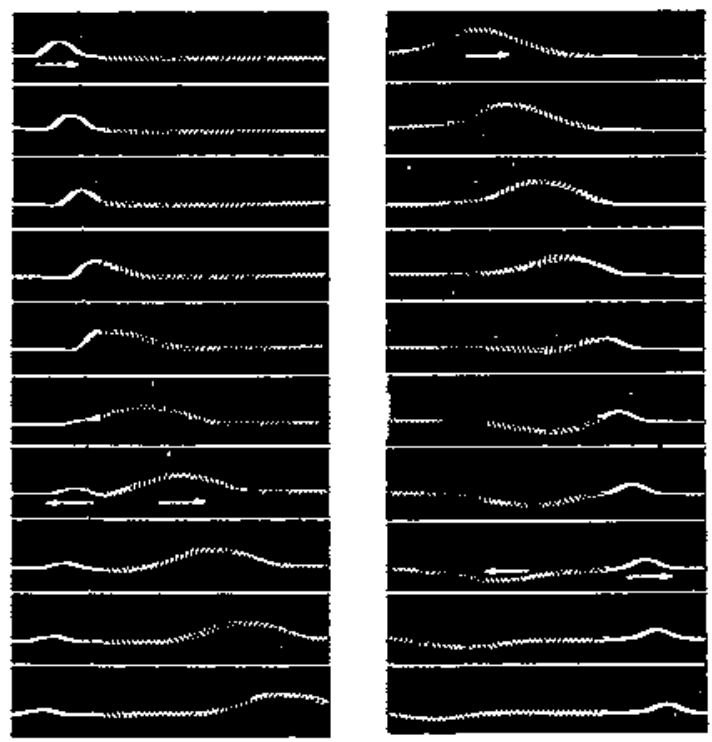

Fig. 20-16. (ndas incidente, reflejada y transmitida a lo largo de dos cuerdas de densidad linear atadas juntas. En (b) y (d) la cuerda que transporta la onda incidente es más pesada; en (c) y (e) la cuerda de la izquierda es más ligera.

ral de una señal en una cuerda (Lacourt 1982, Martin 1983): consiste en una tablilla sobre la que se han fijado tres clavos, un cordel se destiza sobre los tres clavos y durante el desplazamiento de la tablilla se simula la propagación de una señal. La velocidad de desplazamiento está fijada por el operador y la anchura de la "señal", fijada por la separación de dos clavos, es independiente de esta velocidad.

- El problema que se plantea es saber si es necesario utilizar un dispositivo de este tipo, ya que la descripción espacio-temporal de una señal es la del movimiento rectilíneo y uniforme de una forma extendida que puede ser también la de un sólido, de un coche por ejemplo.
- Hallamos en la mayoría de autores de manuales Ia dificultad de expresar las no-dependencias funcionales interesantes que apunta Rozier (1984) en otro campo. Parece que piensen que basta decir a los estudiantes que la velocidad de propagación de una señal en un medio dado es constante para que lo entiendan y lo asimilen. Los resultados obtenidos durante estas distintas encuestas demuestran la insuficiencia de un método como ése. Sería juicioso explicitar los factores de los que depende la velocidad de propagación, pero también aquéllos de los que no depende. Preguntas cualitativas como las que hemos formulado para esta investigación, experiencias que incitan a hacer previsiones y que juegan con la diferencia que hay entre los valores de una misma magnitud en dos situaciones idénticas, por otro lado, permitirían a los estudiantes aprender a eliminar los parámetros no pertinentes en los que han pensado.

- Para luchar contra la tendencia a la numerización de las relaciones y acostumbrar a los estudiantes a usarlas funcionaimente, sería juicioso presentarles ejercicios cualitativos. En efecto, como la gran mayoría de los ejercicios propuestos en la enseñanza actual son cuantitativos (Saltiel 1989), los alumnos utilizan las relaciones aprendidas únicamente como medio de cálculo. Habría que hacer variar, en una situación dada, cada magnitud, una por una, explicitando las que siguen constantes, y mostrarles que procedimientos de reducción funcional llevan a contradicciones.

\section{NOTAS}

1. Se interrogó a 1.300 estudiantes: 700 no habian recibido ninguna enșeñanza sobre ondas: alumnos de enseñanza secundaria francesa, de edades comprendidas entre 15 y 18 años; 600 habian recibido esa enseñanza: alumnos de la secundaria cientifica, estudiantes de primer o segundo curso de universidad en París 7 o en Gembloux (Bélgica), de una escuela superior francesa (E.N.S. de Cachan, especialidad de Física, de 3 a 5 años después de la selectividad).

\section{El subrayado es nuestro.}

3. Esta protuberancia era la que tenía la amplitud mayor y la mayor anchura. Uno de las otras dos protuberancias tenía una amplitud dos veces más pequeña y una anchura idéntica. La úlitima protuberancia tenf́a una ampitiud idéntica y una anchura dos veces menor.

4. Nota del autor. 


\section{REFERENCIAS BIBLIOGRÁFICAS}

ALONSON y FINN, 1977.Physique générale, Champset Ondes, Vol. 2, (Interéditions), pp. 381 .

CLOSSET, J.L., 1989. Les obstacles à l'aprrentissage de l'electrocinétique, B.U.P., 716, París, pp. 931-949.

LACOURT, J., 1982. Physique de Première Set E, pp. 148. (A. Colin: París).

MARTIN, P., 1983. Enregistrement du monvement d'un point affecté par un ébranlement transversal, B.U.P., 652, París, pp. 699 .

MAURINES, L. y SALTIEL, E., 1988. Mécanique spontanée du signal, B.U.P., 707, pp. 1023-1042.

PIAGET, J., 1972. Études d'épistémologie gênétique: Vol. 20, Epistémologie du temps; Vol. 21, Perception et notion de temps, (P.U.F.: París).

ROZIER, S., 1984. Les étudiants et les fonctions de plusieurs variables. Actes de l'école d'été de didactique des mathématiques. (I.R.E.M. de l'Université Paris 7).
ROZIER, S. y VIENNOT, L., 1990. Students ${ }^{\dagger}$ reasoning in thermodynamics, to be published, European Journal of Science Education.

SALTIEL, E. y MALGRANGE, I.L., 1980. Spontaneous ways of reasoning in elementary kinematics, European Journal of Physics, Vol. 1, p. 73.

SALTIEL, E., 1989. Les exercices qualitatifs fonctionnels, colloque sur les finalités des enseignements scientifiques, Marseille, diffusé par le Groupe de Recherche en Didactique de la Physique, pp. 113-122.

VIENNOT, L., 1979. Spontaneous reasoning in elementary dynamics, European Journal of Science Education, Vol, 1(2), pp. 205-221.

VIENNOT, L., 1982. Limplicite en physique: les étudiants et les constantes, European Journal of Physics, Vol. 3, pp. 174-180.

VIENNOT, L., 1989. Tendance à la réduction fonctionnelle: obstacle épistémologique et conflit socio-cognitifs, pp. 8492. (Montréal, Ed: Agence d'ARC inc. Ottawa). 